\title{
Non-local meta-conformal invariance in diffusion-limited erosion
}

\author{
Malte Henkel ${ }^{a, b}$ \\ ${ }^{a}$ Rechnergestützte Physik der Werkstoffe, Institut für Baustoffe (IfB), \\ ETH Zürich, Stefano-Franscini-Platz 3, CH - 8093 Zürich, Switzerland \\ ${ }^{b}$ Groupe de Physique Statistique, Département de Physique de la Matière et des Matériaux, \\ Institut Jean Lamour (CNRS UMR 7198), Université de Lorraine Nancy, \\ B.P. 70239, F - 54506 Vandouvre lès Nancy Cedex, France1
}

\begin{abstract}
The non-stationary relaxation and physical ageing in the diffusion-limited erosion process (DLE) is studied through the exact solution of its Langevin equation, in $d$ spatial dimensions. The dynamical exponent $z=1$, the growth exponent $\beta=\max (0,(1-d) / 2)$ and the ageing exponents $a=b=d-1$ and $\lambda_{C}=\lambda_{R}=d$ are found. In $d=1$ spatial dimension, a new representation of the meta-conformal Lie algebra, isomorphic to $\mathfrak{s l}(2, \mathbb{R}) \oplus \mathfrak{s l}(2, \mathbb{R})$, acts as a dynamical symmetry of the noise-averaged DLE Langevin equation. Its infinitesimal generators are non-local in space. The exact form of the full time-space dependence of the two-time response function of DLE is reproduced for $d=1$ from this symmetry. The relationship to the terrace-step-kink model of vicinal surfaces is discussed.
\end{abstract}

Keywords: diffusion-limited erosion, laplacian growth, physical ageing, conformal invariance, local scale-invariance, non-locality, terrace-step-kink model PACS numbers: 05.40.Fb, 05.10.Gg, 81.10.Aj, 11.25.Hf

\footnotetext{
${ }^{1}$ permanent address
} 
1. The physics of the growth of interfaces is a paradigmatic example of the emergence of non-equilibrium phenomena cooperative phenomena [2, 28, 8, 41, 15]. The most common universality classes, such as the Edwards-Wilkinson (EW) [11], Kardar-Parisi-Zhang (KPZ) [24, Wolf-Villain (WV) 45] or Arcetri [21] classes, are usually specified in terms of models describing the deposition of particles on a surface, leading to the formation of a fluctuating height profile $h(t, \boldsymbol{r})$ of the interface. The cooperative nature of the phenomenon is expressed in the long-time Family-Viscek scaling behaviour [12] of the interface width

$$
w^{2}(t ; L):=\frac{1}{L^{d}} \sum_{\boldsymbol{r} \in \mathscr{L}}\langle(h(t, \boldsymbol{r})-\bar{h}(t))\rangle^{2}=L^{2 \alpha} f_{w}\left(t L^{-z}\right) \sim \begin{cases}t^{2 \beta} & ; \quad \text { if } t L^{-z} \ll 1 \\ L^{2 \alpha} & ; \text { if } t L^{-z} \gg 1\end{cases}
$$

on a hyper-cubic lattice $\mathscr{L} \subset \mathbb{Z}^{d}$ of $|\mathscr{L}|=L^{d}$ sites, where $\langle$.$\rangle denotes an average over many$ independent samples and $\bar{h}(t):=L^{-d} \sum_{\boldsymbol{r} \in \mathscr{L}} h(t, \boldsymbol{r})$ is the spatially averaged height. Herein, $\alpha$ is the roughness exponent, $\beta$ the growth exponent and $z=\alpha / \beta>0$ the dynamical exponent. The interface is called rough if $\beta>0$ and smooth if $\lim _{t \rightarrow \infty} w(t)$ is finite. While theoretical studies abound, reliable experimental results are quite recent. Examples for the KPZ class include turbulent liquid crystals, cell colony growth, collö̈ds, paper combustion, auto-catalytic reaction fronts, thin semiconductor films and sedimentation-electrodispersion, see [43, 15] for recent reviews and [21] for a list of measured values of these exponents. More subtle aspects can be studied through the non-equilibrium relaxation, analogous to physical ageing e.g. in glasses or simple magnets [18]. Analysis proceeds via the two-time correlator $C(t, s ; \boldsymbol{r})$ and the two-time response $R(t, s ; \boldsymbol{r})$. For sufficiently large lattices (where effectively $L \rightarrow \infty$ ) one expects, in the long-time scaling limit $t, s \rightarrow \infty$ with $y:=t / s>1$ fixed, the scaling behaviour

$$
\begin{aligned}
C(t, s ; \boldsymbol{r}):=\langle(h(t, \boldsymbol{r})-\langle\bar{h}(t)\rangle)(h(s, \mathbf{0})-\langle\bar{h}(s)\rangle)\rangle=s^{-b} F_{C}\left(\frac{t}{s} ; \frac{\boldsymbol{r}}{s^{1 / z}}\right) \\
R(t, s ; \boldsymbol{r}):=\left.\frac{\delta\langle h(t, \boldsymbol{r})-\bar{h}(t)\rangle}{\delta j(s, \mathbf{0})}\right|_{j=0}=\langle h(t, \boldsymbol{r}) \widetilde{h}(s, \mathbf{0})\rangle=s^{-1-a} F_{R}\left(\frac{t}{s} ; \frac{\boldsymbol{r}}{s^{1 / z}}\right)
\end{aligned}
$$

where spatial translation-invariance has been implicitly admitted and $j$ is an external field conjugate 1 to $h$. The autocorrelation exponent $\lambda_{C}$ and the autoresponse exponent $\lambda_{R}$ are defined from the asymptotics $F_{C, R}(y, \mathbf{0}) \sim y^{-\lambda_{C, R} / z}$ as $y \rightarrow \infty$. For these non-equilibrium exponents, one has $b=-2 \beta$ and the bound $\lambda_{C} \geq(d+z b) / 2$. For the EW, KPZ, WV and Arcetri classes, where the dynamical exponent $z \geq \frac{3}{2}$, the values of $a, b, \lambda_{C}, \lambda_{R}$ have been determined either analytically or in simulations [29, 36, 10, 17, 9, 20, 14, 32, 21] or else experimentally [42].

Here, we shall be interested in a different universality class, namely diffusion-limited erosion (DLE) [26], often also referred to as Laplacian growth. We shall first derive the width $w(t)$, the correlator $C(t, s ; \boldsymbol{r})$ and the response $R(t, s ; \boldsymbol{r})$ from the exact solution of the defining Langevin equation. Then, for $d=1$ spatial dimension, we shall construct a new representation of the conformal Lie algebra, in terms of spatially non-local operators. We shall show that (i) this representation acts as a dynamical symmetry of the equation of motion of DLE and (ii) that for $d=1$, this dynamical symmetry (which has $z=1$ ), predicts the form of the response $R(t, s ; \boldsymbol{r}$ ).

2. The DLE process [26] can be defined as a lattice model by considering the diffusive motion of a corrosive particle, which starts initially far away from the interface. When the particle finally reaches the interface, it erodes a particle from that interface. Repeating this process

\footnotetext{
${ }^{1}$ In the context of Janssen-de Dominicis theory, $\widetilde{h}$ is the conjugate response field to $h$, see [41].
} 
many times, an eroding interface forms which is described in terms of a fluctuating height $h(t, \boldsymbol{r})$. This leads to the Langevin equation for $h(t, \boldsymbol{r})$ in DLE. In Fourier space [26, 27]

$$
\partial_{t} \widehat{h}(t, \boldsymbol{q})=-\nu|\boldsymbol{q}| \widehat{h}(t, \boldsymbol{q})+\widehat{\jmath}(t, \boldsymbol{q})+\widehat{\eta}(t, \boldsymbol{q})
$$

including the gaussian white nois $2^{2} \widehat{\eta}$, with the variance $\left\langle\widehat{\eta}(t, \boldsymbol{q}) \widehat{\eta}\left(t^{\prime}, \boldsymbol{q}^{\prime}\right)\right\rangle=2 \nu T \delta\left(t-t^{\prime}\right) \delta\left(\boldsymbol{q}+\boldsymbol{q}^{\prime}\right)$ and the constants $\nu, T$ and an external perturbation $\widehat{\jmath}$. Several lattice formulations of the model exist [26, 44, 1, 46]. Flat and radial geometries are compared in [16]. Potential applications of DLE may include contact lines of a liquid meniscus and crack propagation [30]. Remarkably, for $d=1$ space dimension, the Langevin equation (44) has been argued [38] to be related to a system of non-interacting fermions, conditioned to an a-typically large flux. Consider the terrace-stepkink model of a vicinal surface, and interpret the steps as the world lines of fermions. Its transfer matrix is the matrix exponential of the quantum hamiltonian $H$ of the asymmetric XXZ chain [38]. Use Pauli matrices $\sigma_{n}^{ \pm, z}$, attached to each site $n$, such that the particle number at each site is $\varrho_{n}=\frac{1}{2}\left(1+\sigma_{n}^{z}\right)=0,1$. On a chain of $N$ sites [38, 35, 25]

$$
H=-\frac{w}{2} \sum_{n=1}^{N}\left[2 v \sigma_{n}^{+} \sigma_{n+1}^{-}+2 v^{-1} \sigma_{n}^{-} \sigma_{n+1}^{+}+\Delta\left(\sigma_{n}^{z} \sigma_{n+1}^{z}-1\right)\right]
$$

where $w=\sqrt{p q} e^{\mu}, v=\sqrt{p / q} e^{\lambda}$ and $\Delta=2(\sqrt{p / q}+\sqrt{q / p}) e^{-\mu}$. Herein, $p, q$ describe the left/right bias of single-particle hopping and $\lambda, \mu$ are the grand-canonical parameters conjugate to the current and the mean particle number. In the continuum limit, the particle density $\varrho_{n}(t) \rightarrow \varrho(t, r)=\partial_{r} h(t, r)$ is related to the height $h$ which in turn obeys (44), with a gaussian white noise $\eta$ [38]. This follows from the application of the theory of fluctuating hydrodynamics, see [39, 6] for recent reviews. The low-energy behaviour of $H$ yields the dynamical exponent $z=1$ [38, 35, 25]. 3 If one conditions the system to an a-typically large current, the largetime, large-distance behaviour of (5) has very recently been shown [25] (i) to be described by a conformal field-theory with central charge $c=1$ and (ii) the time-space scaling behaviour of the stationary structure function has been worked out explicitly, for $\lambda \rightarrow \infty$. Therefore, one may conjecture that the so simple-looking eq. (4) should furnish an effective continuum description of the large-time, long-range properties of quite non-trivial systems, such as (5)).

The solution of (4) reads in momentum space

$$
\widehat{h}(t, \boldsymbol{q})=e^{-\nu|\boldsymbol{q}| t} \widehat{h}(0, \boldsymbol{q})+\int_{0}^{t} \mathrm{~d} \tau e^{-\nu|\boldsymbol{q}|(t-\tau)}(\widehat{\jmath}(\tau, \boldsymbol{q})+\widehat{\eta}(\tau, \boldsymbol{q}))
$$

In this letter, we focus on the non-equilibrium relaxation of DLE, starting from an an initially flat interface $h(0, \boldsymbol{r})=0$. If $\widehat{\jmath}(t, \boldsymbol{q})=0$, the average interface position remains fixed, thus $\langle\widehat{h}(t, \boldsymbol{q})\rangle=0$ and $\langle h(t, \boldsymbol{r})\rangle=0$. The two-time correlator and response are

$$
\begin{aligned}
& \widehat{C}\left(t, s ; \boldsymbol{q}, \boldsymbol{q}^{\prime}\right):=\left\langle\widehat{h}(t, \boldsymbol{q}) \widehat{h}\left(s, \boldsymbol{q}^{\prime}\right)\right\rangle=\frac{T}{|\boldsymbol{q}|}\left[e^{-\nu|\boldsymbol{q}||t-s|}-e^{-\nu|\boldsymbol{q}|(t+s)}\right] \delta\left(\boldsymbol{q}+\boldsymbol{q}^{\prime}\right) \\
& \widehat{R}\left(t, s ; \boldsymbol{q}, \boldsymbol{q}^{\prime}\right):=\left.\frac{\delta\langle\widehat{h}(t, \boldsymbol{q})\rangle}{\delta \widehat{\jmath}\left(s, \boldsymbol{q}^{\prime}\right)}\right|_{j=0}=\Theta(t-s) e^{-\nu|\boldsymbol{q}|(t-s)} \delta\left(\boldsymbol{q}+\boldsymbol{q}^{\prime}\right)
\end{aligned}
$$

\footnotetext{
${ }^{2}$ Below, we shall refer to (4) with $\widehat{\eta}=0$ as the deterministic part of (4).

${ }^{3}$ Empirically, the bubbles in the price of crude oil display dynamical scaling of the form (1) with $z \approx 1$ [13].
} 
which becomes in direct space, with $\mathcal{C}_{0}:=\pi^{-(d+1) / 2} \Gamma((d+1) / 2) / \Gamma(d / 2)$, and for $d \neq 1$

$$
\begin{aligned}
& C(t, s ; \boldsymbol{r})=\frac{T \mathcal{C}_{0}}{d-1}\left[\left(\nu^{2}(t-s)^{2}+r^{2}\right)^{-(d-1) / 2}-\left(\nu^{2}(t+s)^{2}+r^{2}\right)^{-(d-1) / 2}\right] \\
& R(t, s ; \boldsymbol{r})=\mathcal{C}_{0} \Theta(t-s) \nu(t-s)\left(\nu^{2}(t-s)^{2}+r^{2}\right)^{-(d+1) / 2}
\end{aligned}
$$

where the Heaviside function $\Theta$ expresses the causality condition $t>s$. In particular, the interface width $w^{2}(t)=C(t, t ; \mathbf{0})$ is (apply a high-momentum cut-off $\Lambda$ for $L \rightarrow \infty$, if $d>1$ )

$$
w^{2}(t)=\frac{T \mathcal{C}_{0}}{1-d}\left[(2 \nu t)^{1-d}-\mathcal{C}_{1}(\Lambda)\right] \stackrel{t \rightarrow \infty}{\simeq} \begin{cases}T \mathcal{C}_{0} \mathcal{C}_{1}(\Lambda) /(d-1) & ; \text { if } d>1 \\ T \mathcal{C}_{0} \ln (2 \nu t) & ; \text { if } d=1 \\ {\left[T \mathcal{C}_{0}(2 \nu)^{1-d} /(1-d)\right] \cdot t^{1-d}} & ; \text { if } d<1\end{cases}
$$

This shows the upper critical dimension $d^{*}=1$ of DLE, such that at late times the interface is smooth for $d>1$ and rough for $d \leq 1$ [26]. In the long-time stationary limit $t, s \rightarrow \infty$ with the time difference $\tau=t-s$ being kept fixed, one has the fluctuation-dissipation relationship $\partial C(s+\tau, s ; \boldsymbol{r}) / \partial \tau=-\nu T R(s+\tau, s ; \boldsymbol{r})$. This was to be expected, since there exist lattice model versions in the DLE class which can be formulated in terms of an equilibrium system [44. Finally, in the long-time scaling limit $t, s \rightarrow \infty$ with $y:=t / s>1$ being kept fixed, one may read off from (8) the exponents

$$
\beta=\alpha=\left\{\begin{array}{ll}
0 & ; \text { if } d>1 \\
(1-d) / 2 & ; \text { if } d<1
\end{array} \quad, \quad z=1 \quad, \quad a=b=d-1, \quad \lambda_{C}=\lambda_{R}=d\right.
$$

In contrast to the interface width $w(t)$, which shows a logarithmic growth at $d=d^{*}=1$, logarithms cancel in the two-time correlator $C$ and response $R$, up to additive logarithmic corrections to scaling. This is well-established in the physical ageing of magnetic systems [18].

3. Can one explain the form of the two-time scaling functions of the DLE in terms of a dynamical symmetry ? Such an approach, based on extensions of the dynamical scaling $t \mapsto b^{z} t$ and $\boldsymbol{r} \mapsto b \boldsymbol{r}$ to a larger set of transformations where $b=b(t, \boldsymbol{r})$ becomes effectively time-space-dependent, has been applied and tested in the physical ageing of magnetic systems, quenched either to their critical temperature $T=T_{c}>0$ or else to $T<T_{c}$ (where $z=2$ ), see [18] for a detailed review. More recently, this was also done for the relaxation dynamics in interface growth, namely for the the EW class [36] where $z=2$ and the $(1+1) D$ KPZ class [20], where $z=\frac{3}{2}$. These tests mainly involved the fitting of the auto-response $R(t, s ; \mathbf{0})$ to the exact solutions or the numerical data. Since in the DLE class, one has $z=1$, a different set of local time-space transformations must be sought. It might look tempting to consider conformal invariance [5], well-known from equilibrium critical phenomena, by simply relabelling one of the spatial directions as 'time', since this would give $z=1$. However, as we shall see, a more precise definition is needed. For notational simplicity, we now restrict to the case of $1+1$ time-space dimensions, labelled by a 'time coordinate' $t$ and a 'space coordinate' $r$.

Definition. 1. A set of ortho-conformal transformations (usually called 'conformal transformation') $\mathscr{O}$ is a set of maps $(t, r) \mapsto\left(t^{\prime}, r^{\prime}\right)=\mathscr{O}(t, r)$ of local coordinate transformations, depending analytically on several parameters, such that angles in the coordinate space of the points $(t, r)$ are kept invariant. The maximal finite-dimensional Lie sub-algebra of ortho-conformal transformations is isomorphic to $\mathfrak{c o n f}(2) \cong \mathfrak{s l}(2, \mathbb{R}) \oplus \mathfrak{s l}(2, \mathbb{R})$. A physical system is ortho-conformally invariant if its n-point functions transform covariantly under ortho-conformal transformations. 2. A set of meta-conformal transformations $\mathscr{M}$ is a set of maps $(t, r) \mapsto\left(t^{\prime}, r^{\prime}\right)=\mathscr{M}(t, r)$, de-

\footnotetext{
${ }^{4}$ From the greek prefix $o \varrho \theta o$ : right, standard.

${ }^{5}$ From the greek prefix $\mu \varepsilon \tau \alpha$ : of secondary rank.
} 
pending analytically on several parameters, whose maximal finite-dimensional Lie sub-algebra of meta-conformal transformations is isomorphic to conf(2). A physical system is meta-conformally invariant if its n-point functions transform covariantly under meta-conformal transformations.

Hence, ortho-conformal transformations are also meta-conformal transformations.

In $(1+1) D$, ortho-conformal transformations are all analytic or anti-analytic maps, $z \mapsto f(z)$ or $\bar{z} \mapsto \bar{f}(\bar{z})$, of the complex variables $z=t+\mathrm{i} r, \bar{z}=t-\mathrm{i} r$. For our purposes, we restrict here to the projective conformal transformations $z \mapsto \frac{\alpha z+\beta}{\gamma z+\delta}$ with $\alpha \delta-\beta \gamma=1$ and similarly for $\bar{z}$. Then the Lie algebra generators $\ell_{n}=-z^{n+1} \partial_{z}$ and $\bar{\ell}_{n}=-\bar{z}^{n+1} \partial_{\bar{z}}$ with $n= \pm 1,0$ span the Lie algebra $\operatorname{conf}(2) \cong \mathfrak{s l}(2, \mathbb{R}) \oplus \mathfrak{s l}(2, \mathbb{R})$. We shall use below the basis $X_{n}:=\ell_{n}+\bar{\ell}_{n}$ and $Y_{n}:=\ell_{n}-\bar{\ell}_{n}$. In an ortho-conformally invariant physical system, these generators act on physical 'quasi-primary' [5] scaling operators $\phi=\phi(z, \bar{z})=\varphi(t, r)$ and then contain also terms which describe how these quasi-primary operators should transform, namely

$$
\ell_{n}=-z^{n+1} \partial_{z}-\Delta(n+1) z^{n}, \bar{\ell}_{n}=-\bar{z}^{n+1} \partial_{\bar{z}}-\bar{\Delta}(n+1) \bar{z}^{n}
$$

where $\Delta, \bar{\Delta}$ are the conformal weights of the scaling operator $\phi$. Laplace's equation $\mathcal{S} \phi=$ $4 \partial_{z} \partial_{\bar{z}} \phi=0$ is a simple example of an ortho-conformally invariant system, since the commutator

$$
\left[\mathcal{S}, \ell_{n}\right] \phi(z, \bar{z})=-(n+1) z^{n} \mathcal{S} \phi(z, \bar{z})-4 \Delta n(n+1) z^{n-1} \partial_{\bar{z}} \phi(z, \bar{z})
$$

shows that for a scaling operator $\phi$ with $\Delta=\bar{\Delta}=0$, the space of solutions of the Laplace equation $\mathcal{S} \phi=0$ is conformally invariant, since any solution is mapped onto another solution in the transformed coordinates $7 \mathrm{~A}$ two-point function of quasi-primary scaling operators is $\mathscr{C}\left(t_{1}, t_{2} ; r_{1}, r_{2}\right):=\left\langle\phi_{1}\left(z_{1}, \bar{z}_{1}\right) \phi_{2}\left(z_{2}, \bar{z}_{2}\right)\right\rangle=\left\langle\varphi_{1}\left(t_{1}, r_{1}\right) \varphi_{2}\left(t_{2}, r_{2}\right)\right\rangle$. Its covariance under orthoconformal transformations is expressed by the 'projective Ward identities' $X_{n} \mathscr{C}=Y_{n} \mathscr{C}=0$ for $n= \pm 1,0$ [5]. For scalars, such that $\Delta_{i}=\bar{\Delta}_{i}=x_{i}$, this gives [34]

$$
\mathscr{C}\left(t_{1}, t_{2} ; r_{1}, r_{2}\right)=\mathcal{C}_{0} \delta_{x_{1}, x_{2}}\left(\left(t_{1}-t_{2}\right)^{2}+\left(r_{1}-r_{2}\right)^{2}\right)^{-x_{1}}
$$

where $\mathcal{C}_{0}$ is a normalisation constant.

An example of meta-conformal transformations in $(1+1)$ dimensions is given by [17]

$$
\begin{aligned}
X_{n} & =-t^{n+1} \partial_{t}-\mu^{-1}\left[(t+\mu r)^{n+1}-t^{n+1}\right] \partial_{r}-(n+1) x t^{n}-(n+1) \frac{\gamma}{\mu}\left[(t+\mu r)^{n}-t^{n}\right] \\
Y_{n} & =-(t+\mu r)^{n+1} \partial_{r}-(n+1) \gamma(t+\mu r)^{n}
\end{aligned}
$$

where $x, \gamma$ are the scaling dimension and the 'rapidity' of the scaling operator $\varphi=\varphi(t, r)$ on which these generators act and the constant $1 / \mu$ has the dimensions of a velocity. The Lie algebra $\left\langle X_{n}, Y_{n}\right\rangle_{n= \pm 1,0}$ is isomorphic to $\operatorname{con} \mathfrak{f}(2)$ [22]. An invariant equation 8 is $\mathcal{S} \varphi=\left(-\mu \partial_{t}+\right.$ $\left.\partial_{r}\right) \varphi=0$, provided only that $\gamma=\mu x$, since the only non-vanishing commutators of the Lie algebra with $\mathcal{S}$ are $\left[\mathcal{S}, X_{0}\right] \varphi=-\mathcal{S} \varphi$ and $\left[\mathcal{S}, X_{1}\right] \varphi=-2 t \mathcal{S} \varphi+2(\mu x-\gamma) \varphi$. The covariant two-point function is [17, 23]

$$
\mathscr{C}\left(t_{1}, t_{2} ; r_{1}, r_{2}\right)=\mathcal{C}_{0} \delta_{x_{1}, x_{2}} \delta_{\gamma_{1}, \gamma_{2}}\left(t_{1}-t_{2}\right)^{-2 x_{1}}\left(1+\frac{\mu}{\gamma_{1}}\left|\gamma_{1} \frac{r_{1}-r_{2}}{t_{1}-t_{2}}\right|\right)^{-2 \gamma_{1} / \mu}
$$

\footnotetext{
${ }^{6}$ Interpretation: $X_{-1}, Y_{-1}$ generate time- and space-translations, $X_{0}$ global dilatations $t \mapsto b t, r \mapsto b r, Y_{0}$ rigid time-space rotations and $X_{1}, Y_{1}$ generate the 'special' conformal transformations.

${ }^{7}$ This concept of a dynamical symmetry, for the free diffusion equation, goes back to Jacobi (1842) and Lie (1881) and was re-introduced into physics by Niederer (1972) [31.

${ }^{8}$ See [40] for extensions as dynamical symmetries of the $(1+1) D$ Vlassov equation, isomorphic to conf $(2)$.
} 
Table 1: Comparison of ortho- and two examples of meta-conformal invariance. Listed are the commutators of the Lie algebra bases $\left\langle X_{n}, Y_{n}\right\rangle_{n= \pm 1,0} \cong \mathfrak{c o n f}(2)$, the invariant Schrödinger operator $\mathcal{S}$ and the covariant two-point function $\mathscr{C}(t ; r)=\langle\varphi(t, r) \varphi(0,0)\rangle$, up to normalisation. The physical nature of $\mathscr{C}$ is also indicated.

For ortho-conformal invariance and meta-conformal invariance 1 , one has the constraints $x_{1}=$ $x_{2}$ and $\gamma_{1}=\gamma_{2}$. For the meta-conformal invariance 2, we list only case $\mathrm{A}$ from the text. One has $\mu^{-1}=\mathrm{i} \nu$ with $\nu>0$, and the constraints $\gamma_{1}+\gamma_{2}=\mu$ and $\gamma_{1}-\gamma_{2}=\mu\left(x_{1}-x_{2}\right)$.

\begin{tabular}{|c|c|c|c|}
\hline & ortho & meta-1 & meta-2 \\
\hline $\begin{array}{l}\text { Lie } \\
\text { algebra } \\
\operatorname{conf}(2)\end{array}$ & $\begin{array}{l}{\left[X_{n}, X_{m}\right]=(n-m) X_{n+m}} \\
{\left[X_{n}, Y_{m}\right]=(n-m) Y_{n+m}} \\
{\left[Y_{n}, Y_{m}\right]=(n-m) X_{n+m}}\end{array}$ & $\begin{array}{l}{\left[X_{n}, X_{m}\right]=(n-m) X_{n+m}} \\
{\left[X_{n}, Y_{m}\right]=(n-m) Y_{n+m}} \\
{\left[Y_{n}, Y_{m}\right]=\mu(n-m) Y_{n+m}}\end{array}$ & $\begin{array}{l}{\left[X_{n}, X_{m}\right]=(n-m) X_{n+m}} \\
{\left[X_{n}, Y_{m}\right]=(n-m) Y_{n+m}} \\
{\left[Y_{n}, Y_{m}\right]=\mu(n-m) Y_{n+m}}\end{array}$ \\
\hline $\mathcal{S}$ & $\partial_{t}^{2}+\partial_{r}^{2}$ & $-\mu \partial_{t}+\partial_{r}$ & $-\mu \partial_{t}+\nabla_{r}$ \\
\hline $\mathscr{C}$ & $\begin{array}{l}\left(t^{2}+r^{2}\right)^{-x_{1}} \\
\text { correlator }\end{array}$ & $\begin{array}{l}t^{-2 x_{1}}\left(1+\frac{\mu}{\gamma_{1}}\left|\frac{\gamma_{1} r}{t}\right|\right)^{-2 \gamma_{1} / \mu} \\
\text { correlator }\end{array}$ & $\begin{array}{l}t^{1-x_{1}-x_{2}} \cdot \nu t\left(\nu^{2} t^{2}+r^{2}\right)^{-1} \\
\text { response } \quad(\text { case } \mathrm{A})\end{array}$ \\
\hline
\end{tabular}

These well-known results are summarised in the first two columns of table 1. Comparing the two-point functions (13) and (15) shows that even for the same dynamical exponent $z=1$, different forms of the scaling functions are possible for ortho- and meta-conformal invariance.

4. Are these examples of ortho- or meta-conformal invariance, which have $z=1$ and are realised in terms of local first-order differential operators, suitable as a dynamical symmetry of the DLE in $1+1$ dimensions ? This must be answered in the negative, for the following reasons.

1. The DLE response function (8b) is distinct from the predictions (13) 15 , see also table 1 , For the meta-conformal two-point function (15), the functional form is clearly different for finite values of the scaling variable $v=\left(r_{1}-r_{2}\right) /\left(t_{1}-t_{2}\right)$. The ortho-conformal twopoint function (13) looks to be much closer, with the choice $x_{1}=\frac{1}{2}$ and the scale factor fixed to $\nu=1$, were it not for the extra factor $\nu(t-s)$. On the other hand, the two-time DLE correlator (8a) does not agree with (13) either, but might be similar to a two-point function computed in a semi-infinite space $t \geq 0, r \in \mathbb{R}$ with a boundary at $t=0$.

2. The invariant equations $\mathcal{S} \varphi=0$ are distinct from the deterministic part of the DLE Langevin equation (4). Recall the well-known fact [33] that for Langevin equations $\mathcal{S} \varphi=$ $\eta$, where $\eta$ is a white noise, and where the noise-less equation $\mathcal{S} \varphi_{0}=0$ has a local scaleinvariance (including a generalised Galilei-invariance to derive Bargman super-selection rules [3]) all correlators and response functions can be reduced to responses found in the noise-less theory. In particular, the two-time response function of the full noisy equation $R(t, s ; \boldsymbol{r})=R_{0}(t, s ; \boldsymbol{r})$, is identical to the response $R_{0}$ found when the noise is turned off and computed from the dynamical symmetry [33, 18].

Indeed, in the example (7b $8 \mathrm{~b})$ of the DLE, one sees that the two-time response $R$ is independent of $T$, which characterises the white noise.

We shall look for dynamical symmetries of the equation $\mathcal{S} \varphi=\left(-\mu \partial_{t}+\nabla_{r}\right) \varphi=0$, which is the 
deterministic part of the DLE Langevin equation (44), in $1+1$ dimensions. We shall seek to derive the form of the two-time response function $R(t, s ; \boldsymbol{r})$ from this dynamical symmetry. The twotime correlator $C$ cannot be obtained in this way. Rather, we shall see that its 'deterministic' contribution $C_{0}(t, s ; \boldsymbol{r})=0$ simply vanishes. As shown in 33, the correlator must be obtained from an integral over three-point response functions. We leave this for future work.

5. In direct space, the invariant Schrödinger operator for DLE should be $\mathcal{S}:=-\mu \partial_{t}+\nabla_{r}$, where $\nabla_{r}^{\alpha}$ denotes the Riesz-Feller fractional derivative [37] of order $\alpha$. For functions $f(r)$ of a single variable $r \in \mathbb{R}$ (assuming that $f(r)$ is such that the integral exists), we use the convention

$$
\nabla_{r}^{\alpha} f(r):=\frac{\mathrm{i}^{\alpha}}{2 \pi} \int_{\mathbb{R}^{2}} \mathrm{~d} k \mathrm{~d} x|k|^{\alpha} e^{\mathrm{i} k(r-x)} f(x)
$$

Then the following properties hold true, for formal manipulations [4],[18, app. J.2]

$$
\begin{aligned}
\nabla_{r}^{\alpha} \nabla_{r}^{\beta} f(r)=\nabla_{r}^{\alpha+\beta} f(r) \quad, \quad\left[\nabla_{r}^{\alpha}, r\right] f(r)=\alpha \partial_{r} \nabla_{r}^{\alpha-2} f(r), \quad \nabla_{r}^{\alpha} f(\mu r)=|\mu|^{\alpha} \nabla_{\mu r}^{\alpha} f(\mu r) \\
\nabla_{r}^{\alpha} e^{\mathrm{i} q r}=(\mathrm{i}|q|)^{\alpha} e^{\mathrm{i} q r} \quad, \quad\left(\widehat{\nabla_{r}^{\alpha} f(r)}\right)(q)=(\mathrm{i}|q|)^{\alpha} \widehat{f}(q), \quad \nabla_{r}^{2} f(r)=\partial_{r}^{2} f(r)
\end{aligned}
$$

where $\widehat{f}(q)$ is the Fourier transform of $f(r)$. In selecting the generators for the Lie algebra of dynamical symmetries, we follow [17] and require that time translations $X_{-1}=-\partial_{t}$, dilatations $X_{0}=-t \partial_{t}-r \partial_{r}-x$ and space translations $Y_{-1}$ are present. However, if one begins with the standard local generator $-\partial_{r}$ of spatial translations, it turns out that the non-local generator $-\nabla_{r}$ is generated as well [4],[18, ch. 5.3]. The closure of this set of generators, for generic values of $z \neq 2$, is still an open problem. In order to obtain a well-defined Lie algebra of dynamical symmetries of $\mathcal{S}$, we consider a non-local spatial translation operator $Y_{-1}=-\nabla_{r}$. Consider the following set of single-particle generators

$$
\begin{gathered}
X_{-1}=-\partial_{t}, \quad X_{0}=-t \partial_{t}-r \partial_{r}-x, \quad X_{1}=-t^{2} \partial_{t}-2 t r \partial_{r}-\mu r^{2} \nabla_{r}-2 x t-2 \gamma r \partial_{r} \nabla_{r}^{-1}(18) \\
Y_{-1}=-\nabla_{r}, \quad Y_{0}=-t \nabla_{r}-\mu r \partial_{r}-\gamma, Y_{1}=-t^{2} \nabla_{r}-2 \mu t r \partial_{r}-\mu^{2} r^{2} \nabla_{r}-2 \gamma t-2 \gamma \mu r \partial_{r} \nabla_{r}^{-1}
\end{gathered}
$$

As in the set (14) of meta-conformal transformations, the constants $x$ and $\gamma$, respectively, are the scaling dimension and rapidity of the scaling operator $\varphi=\varphi(t, r)$ on which these generators act. It is now an afternoon's exercise (before tea time) to check, with the help of (17) 9 the following commutator relations, for $n, m \in\{ \pm 1,0\}$

$$
\left[X_{n}, X_{m}\right]=(n-m) X_{n+m} \quad, \quad\left[X_{n}, Y_{m}\right]=(n-m) Y_{n+m} \quad, \quad\left[Y_{n}, Y_{m}\right]=\mu(n-m) Y_{n+m}
$$

This establishes the Lie algebra isomorphism $\left\langle X_{n}, Y_{n}\right\rangle_{n= \pm 1,0} \cong \mathfrak{c o n f}(2)$. Furthermore, since

$$
\left[\mathcal{S}, Y_{n}\right] \varphi=\left[\mathcal{S}, X_{-1}\right] \varphi=0,\left[\mathcal{S}, X_{0}\right] \varphi=-\mathcal{S} \varphi, \quad\left[\mathcal{S}, X_{1}\right] \varphi=-2 t \mathcal{S} \varphi+2(\mu x-\gamma) \varphi
$$

the infinitesimal transformations (18) form a Lie algebra of meta-conformal dynamical symmetries (of the deterministic part) of the DLE equation (4), if $\gamma=x \mu$. In contrast to the generators (14), the generators (18) are non-local and do not generate simple local changes of the coordinates $(t, r)$. In spite of an attempt to interpret non-local infinitesimal generators as the transformation of a distribution of coordinates [19], finding a clear geometrical interpretation of the generators (18) remains an open problem.

\footnotetext{
${ }^{9}$ Use the identities $\left[\nabla_{r}, r^{2}\right]=2 r \partial_{r} \nabla_{r}^{-1},\left[r^{2} \nabla_{r}, r \partial_{r}\right]=-r^{2} \nabla_{r}$ and $\left[\nabla_{r}, \partial_{r}\right]=\left[\partial_{r} \nabla_{r}^{-1}, r\right]=0$.
} 
6. We look for the covariant $n$-point functions. We expect [18] that these will correspond physically to response functions, i.e. the two-time response $R(t, s ; r)=\langle\varphi(t, r) \widetilde{\varphi}(s, 0)\rangle$, where $\widetilde{\varphi}$ is the response operator conjugate to the scaling operator $\varphi$, in the context of Janssen-de Dominicis theory [41. In order to write down the $n$-body operators analogous to (18), we must ascribe a 'signature' $\varepsilon= \pm 1$ to each scaling operator. We choose the convention that $\varepsilon_{i}=+1$ for scaling operators $\varphi_{i}$ and $\varepsilon_{i}=-1$ for response operators $\widetilde{\varphi}_{i}$. Then

$$
\begin{aligned}
Y_{-1}=Y_{-1}^{[n]} & =\sum_{i}\left[-\varepsilon_{i} \nabla_{i}\right] \quad, \quad Y_{0}=Y_{0}^{[n]}=\sum_{i}\left[-\varepsilon_{i} t_{i} \nabla_{i}-\mu r_{i} D_{i}-\gamma_{i}\right] \\
Y_{1}=Y_{1}^{[n]} & =\sum_{i}\left[-\varepsilon_{i} t_{i}^{2} \nabla_{i}-2 \mu t_{i} r_{i} D_{i}-\mu^{2} \varepsilon_{i} r_{i}^{2} \nabla_{i}-2 \gamma_{i} t_{i}-2 \mu \gamma_{i} \varepsilon_{i} r_{i} D_{i} \nabla_{i}^{-1}\right] \\
X_{-1}=X_{-1}^{[n]} & =\sum_{i}\left[-\partial_{i}\right] \quad, \quad X_{0}=X_{0}^{[n]}=\sum_{i}\left[-t_{i} \partial_{i}-r_{i} D_{i}-x_{i}\right] \\
X_{1}=X_{1}^{[n]} & =\sum_{i}\left[-t_{i}^{2} \partial_{i}-2 t_{i} r_{i} D_{i}-\mu \varepsilon_{i} r_{i}^{2} \nabla_{i}-2 x_{i} t_{i}-2 \gamma_{i} \varepsilon_{i} r_{i} D_{i} \nabla_{i}^{-1}\right]
\end{aligned}
$$

with the short-hands $\partial_{i}=\frac{\partial}{\partial t_{i}}, D_{i}=\frac{\partial}{\partial r_{i}}$ and $\nabla_{i}=\nabla_{r_{i}}$. It can be checked that the generators (21) obey the meta-conformal Lie algebra (19). Now, for a $(n+m)$-point function

$\mathscr{C}_{n, m}=\mathscr{C}_{n, m}\left(t_{1}, \ldots, t_{n+m} ; r_{1}, \ldots, r_{n+m}\right)=\left\langle\varphi_{1}\left(t_{1}, r_{1}\right) \cdots \varphi_{n}\left(t_{n}, r_{n}\right) \widetilde{\varphi}_{n+1}\left(t_{n+1}, r_{n+1}\right) \cdots \widetilde{\varphi}_{n+m}\left(t_{n+m}, r_{n+m}\right)\right\rangle$

of quasi-primary scaling and response operators, the covariance is expressed through the projective Ward identities $X_{k}^{[n+m]} \mathscr{C}_{n, m}=Y_{k}^{[n+m]} \mathscr{C}_{n, m}=0$, for $k= \pm 1,0$.

7. We apply this to the two-time response function $\mathscr{R}=\mathscr{R}\left(t_{1}, t_{2} ; r_{1}, r_{2}\right)=\mathscr{C}_{1,1}\left(t_{1}, t_{2} ; r_{1}, r_{2}\right)$. From $X_{-1} \mathscr{R}=0$ it follows that $\mathscr{R}=\mathscr{R}\left(t ; r_{1}, r_{2}\right)$, with $t=t_{1}-t_{2}$. On the other hand, the condition $Y_{-1} \mathscr{R}=0$ would lead in Fourier space to $\left(\varepsilon_{1}\left|q_{1}\right|+\varepsilon_{2}\left|q_{2}\right|\right) \widehat{\mathscr{R}}\left(t ; q_{1}, q_{2}\right)=0$. Because of the assigned signatures $\varepsilon_{1}=-\varepsilon_{2}=1$, this equation can have a non-vanishing solution such that we can write $\mathscr{R}=F(t, r)$, with $r=r_{1}-r_{2}$. However, for a two-point correlator $\mathscr{C}_{2,0}$, with $\varepsilon_{1}=\varepsilon_{2}=1$, the Ward identity $Y_{-1} \mathscr{C}_{2,0}=0$ would simply imply that $\widehat{\mathscr{C}}_{2,0}=0$, and in agreement with the fact that the DLE-correlator 7 a $18 \mathrm{a}$ ) vanishes as $T \rightarrow 0$. Standard calculations, see e.g. [18], lead to the following set of conditions for the function $\mathscr{R}=F(t, r)$

$$
\begin{aligned}
{\left[-t \partial_{t}-r \partial_{r}-x_{1}-x_{2}\right] F } & =0 \\
{\left[-t \varepsilon_{1} \nabla_{r}-\mu r \partial_{r}-\gamma_{1}-\gamma_{2}\right] F } & =0 \\
{\left[-t^{2} \partial_{t}-2 t r \partial_{r}-\mu r^{2} \varepsilon_{1} \nabla_{r}-2 x_{1} t-2 \gamma_{1} \varepsilon_{1} r \partial_{r} \nabla_{r}^{-1}\right] F } & =0 \\
{\left[-t^{2} \varepsilon_{1} \nabla_{r}-2 \mu t r \partial_{r}-\mu^{2} \varepsilon_{1} r^{2} \nabla_{r}-2 \gamma_{1} t-2 \mu \gamma_{1} \varepsilon_{1} r \partial_{r} \nabla_{r}^{-1}\right] F } & =0
\end{aligned}
$$

Eqs. (22c $\sqrt[22 \mathrm{~d}]{\mathrm{d}})$ can be further simplified by combining them with (22a]|22b) and reduce to

$$
\left(x_{1}-x_{2}\right)\left(t+\mu \varepsilon_{1} r \partial_{r} \nabla_{r}^{-1}\right) F=0, \quad\left(\left(\gamma_{1}-\gamma_{2}\right)-\mu\left(x_{1}-x_{2}\right)\right) t F=0
$$

If $F$ does not contain a factor $\sim \delta(t)$, the second eq. (23) gives the constraint $\gamma_{1}-\gamma_{2}=\mu\left(x_{1}-x_{2}\right)$. Eq. (22a) implies the scaling form $F(t, r)=t^{-2 x} f(v)$, with $v=r / t$ and $x=\frac{1}{2}\left(x_{1}+x_{2}\right)$. From (22b|23), the scaling function $f(v)$ must satisfy, with $\gamma=\frac{1}{2}\left(\gamma_{1}+\gamma_{2}\right)$

$$
\left(\varepsilon_{1} \nabla_{v}+\mu v \partial_{v}+2 \gamma\right) f(v)=0 \quad \text { and } \quad \nabla_{v}^{-1}\left[\left(x_{1}-x_{2}\right)\left(\varepsilon_{1} \nabla_{v}+\mu v \partial_{v}+\mu\right)\right] f(v)=0
$$

The two conditions in eq. (24) are compatible in two distinct cases: 
Case A: $\underline{2 \gamma=\mu}$. Then $\left(\varepsilon_{1} \nabla_{v}+\mu v \partial_{v}+\mu\right) f(v)=0$ and $x_{1} \neq x_{2}$ is still possible.

Case B: $\underline{x}_{1}=x_{2}$. Then $\gamma_{1}=\gamma_{2}$ and $\left(\varepsilon_{1} \nabla_{v}+\mu v \partial_{v}+2 \gamma\right) f(v)=0$.

In Fourier space, eq. (22b) gives $\left(\mathrm{i} \varepsilon_{1}|q|-\mu q \partial_{q}+(2 \gamma-\mu)\right) \widehat{f}(q)=0$, which illustrates the difference between cases $\mathrm{A}$ and B. It follows that $\widehat{f}(q)=\widehat{f}_{0} q^{2 \gamma / \mu-1} \exp \left(\mathrm{i} \varepsilon_{1}|q| / \mu\right)$, where $\widehat{f}_{0}$ is a normalisation constant. Finally, comparison of the Schrödinger operator $\mathcal{S}=-\mu \partial_{t}+\nabla_{r}$ with the DLE equation (4) shows that $\mu^{-1}=\mathrm{i} \nu$. Transforming back into direct space, we find

$$
f(v)=f_{0} \times \begin{cases}\varepsilon_{1} \nu\left(\nu^{2}+v^{2}\right)^{-1} & ; \text { case A } \\ \operatorname{Re}\left(e^{-\mathrm{i} \pi \psi / 2}\left(\varepsilon_{1} \nu-\mathrm{i} v\right)^{-\psi-1}\right) & ; \text { case } \mathrm{B}, \text { with } \psi+1:=2 \mathrm{i} \nu \gamma\end{cases}
$$

A linear combination of these two solutions is a solution of the linear system (24) as well.

8. In particular, for case $\mathrm{A}$, the final form of the two-time response function $\mathscr{R}$ becomes, with a normalisation constant $F_{0}$ and $x=\frac{1}{2}\left(x_{1}+x_{2}\right)$

$$
\mathscr{R}=F(t, r)=F_{0} t^{1-2 x} \frac{\varepsilon_{1} \nu t}{\nu^{2} t^{2}+r^{2}} \quad ; \text { with } t=t_{1}-t_{2}, r=r_{1}-r_{2} \quad \text { (case A) }
$$

If one takes $x=\frac{1}{2}$, and $\nu \in \mathbb{R}_{+}$, this reproduces the exact solution (8b) of the response in $(1+1) D$ DLE. This is our main result: the non-local representation (21) of conf $(2)$ is necessary to reproduce the correct scaling behaviour of the non-stationary response. The properties and predictions of this second example of a meta-conformal symmetry, for the special case A, are listed in the last column of table 1. An important difference is that ortho-conformal invariance and meta-conformal invariance 1 predict the form of a two-time correlator $\mathscr{C}=\mathscr{C}_{2,0}$, whereas the meta-conformal invariance 2 predicts the form of a two-time response $\mathscr{R}=\mathscr{C}_{1,1}$.

Summary: we have proposed a meta-conformal dynamical symmetry for the DLE in $1+1$ dimensions. This symmetry, isomorphic to the Lie algebra $\mathfrak{s l}(2, \mathbb{R}) \oplus \mathfrak{s l}(2, \mathbb{R})$, is realised in terms of non-local generators, see eqs. (18)21). It is distinct from other known representations of the conformal Lie algebra, both in the form of the invariant Schrödinger operator $\mathcal{S}$ and in the predicted shape of the covariant two-point function, see table1. In particular, the generator $Y_{-1}$ which plays the role of 'spatial translations' is manifestly non-local. The full time-space form of the two-time response $R(t, s ; r)$ in $(8 \mathrm{~b})$ can be derived from this dynamical symmetry. This is the first time that (i) the full response (and not only the auto-response $R(t, s ; 0)$ ) can be confirmed and (ii) that the set of generators closes into a Lie algebra, for a system with $z \neq 2$.

In view of Spohn's mapping [38], which relates the $(1+1) D$ DLE equation (44) with the quantum chain (5) and the terrace-step-kink model, a convenient linear combination of the prediction of $R(t, s ; r)$ from cases $\mathrm{A}$ and $\mathrm{B}$ might describe the non-stationary response of vicinal surfaces. Indeed, the explicit form of the connected stationary correlator $\langle\varrho(t, r) \varrho(t, 0)\rangle_{c}$ of the particle density $\varrho(t, r)=\partial_{r} h(t, r)$, obtained by Karevksi and Schütz from (5) in the limit $\lambda \rightarrow \infty$ [25. eq.(1)], contains two terms which look quite analogous to the responses (25) in cases A and $\mathrm{B}$. While that is distinct from the non-stationary responses considered here, the qualitative analogy is encouraging. Certainly, a precise test is called for. This will require to work out higher $n$-point functions in order to be able to derive the form of non-equilibrium correlators. Stationary correlators might be included by considering an appropriate initial condition. An obvious further extension will be to dimensions $d>1$. Conceptually, the consideration of 
manifestly non-local generators in local scale-invariance might lead to further insight for the construction of dynamical symmetries for different values of $z$.

Acknowledgements: I thank G.M. Schütz for fruitful discussions. This work was started at the workshop 'Advanced Conformal Field Theory and Applications' (ACFT) at the Institute Henri Poincaré Paris. It is a pleasure to thank the organisers for their warm hospitality. This work was also partly supported by the Collège Doctoral franco-allemand Nancy-LeipzigCoventry ('Statistical Physics of Complex Systems') of UFA-DFH.

\section{References}

[1] F.D.A. Aarão Reis and J. Stafiej, J. Phys. Cond. Matt. 19, 065125 (2007) [cond-mat/0609428].

[2] A.L. Barabási, H.E. Stanley, Fractal concepts in surface growth, Cambridge University Press (1995).

[3] V. Bargman, Ann. of Math. 56, 1 (1954).

[4] F. Baumann, thèse de doctorat/Dissertation, Nancy \& Erlangen (2007).

[5] A.A. Belavin, A.M. Polyakov and A.B. Zamolodchikov, Nucl. Phys. B241, 333 (1984).

[6] L. Bertini, A. De Sole, D. Gabrielli, G. Jona-Lasinio, C. Landim, Rev. Mod. Phys. 87, 593 (2015) [arXiv:1404.6466].

[7] Y.-L. Chou and M. Pleimling, J. Stat. Mech. P08007 (2010) [arXiv:1007.2380];

Y.-L. Chou and M. Pleimling, Physica A391, 3585 (2012) [arXiv:1112.5867].

[8] I. Corwin, Rand. Matrices Theory Appl. 1, 1130001 (2012) [arXiv:1106.1596].

[9] G.L. Daquila and U.C. Täuber, Phys. Rev. E83, 051107 (2011) [arXiv:1102.2824] .

[10] E. Darvish and A.A. Marsoudi, J. Math. Phys. 50, 013304 (2009).

[11] S.F. Edwards and D.R. Wilkinson, Proc. Roy. Soc. A381, 17 (1982).

[12] F. Family and T. Vicsek, J. Phys. A18, L75 (1985).

[13] S.M. Garcia-Carranco, J. Bory-Reyes, A.S. Balankin, Physica A452, 60 (2016).

[14] T. Halpin-Healy and G. Palansantzas, Europhys. Lett. 105, 50001 (2014) [arXiv:1403.7509].

[15] T. Halpin-Healy and K.A. Takeuchi, J. Stat. Phys. 160, 794 (2015) [arXiv:1505.01910].

[16] L. Hedayatifar, A.A. Masoudi, M. Khorrami, M. Stastna and M. Kohandel, J. Phys. A47, 455003 (2014).

[17] M. Henkel, Nucl. Phys. B641, 405 (2002) [hep-th/0205256].

[18] M. Henkel and M. Pleimling, "Non-equilibrium phase transitions vol. 2: ageing and dynamical scaling far from equilibrium", Springer (Heidelberg 2010).

[19] M. Henkel and S. Stoimenov, Nucl. Phys. B847, 612 (2011) [arXiv:1011.6315].

[20] M. Henkel, J.D. Noh and M. Pleimling, Phys. Rev. E85, 030102(R) (2012) [arXiv:1109.5022]. 
[21] M. Henkel and X. Durang, J. Stat. Mech, P05022 (2015) [arXiv:1501.07745].

[22] M. Henkel, Symmetry 7, 2108 (2015) [arXiv:1509.03669].

[23] M. Henkel and S. Stoimenov, J. Phys. A, at press [arXiv:1607.00685].

[24] M. Kardar, G. Parisi and Y.-C. Zhang, Phys. Rev. Lett. 56, 889 (1986).

[25] D. Karevski and G.M. Schütz, [arXiv:1606.04248].

[26] J. Krug and P. Meakin, Phys. Rev. Lett. 66, 703 (1991).

[27] J. Krug, in A. McKane, M. Droz, J. Vannimenus, D. Wolf (eds) Scale invariance, interfaces and non-equilibrium dynamics, NATO ASI Series vol. B344, Plenum Press (London 1994), p. 1.

[28] J. Krug, Adv. Phys. 46, 139 (1997).

[29] M. Krech, Phys. Rev. E55, 668 (1997) [cond-mat/9609230]; erratum E56, 1285 (1997).

[30] J.F. Joanny and P.G. de Gennes, J. Chem. Phys. 81, 552 (1984);

H. Gao and J.R. Rice, J. Appl. Mech. 56, 828 (1989).

[31] U. Niederer, Helv. Phys. Acta, 45, 802 (1972).

[32] G. Ódor, J. Kelling, S. Gemming, Phys. Rev. E89, 032146 (2014) [arXiv:1312.6029];

J. Kelling, G. Ódor, S. Gemming, Phys. Rev. E94, 022107 (2016) [arXiv:1605.02620] ;

J. Kelling, G. Ódor, S. Gemming, [arXiv:1609.05795].

[33] A. Picone and M. Henkel, Nucl. Phys B688 217, (2004) [arxiv:cond-mat/0402196].

[34] A.M. Polyakov, sov. Phys. JETP Lett. 12, 381 (1970).

[35] V. Popkov and G.M. Schütz, J. Stat. Phys. 142, 627 (2011) [arXiv:1011.3913].

[36] A. Röthlein, F. Baumann and M. Pleimling, Phys. Rev. E74, 061604 (2006) [cond-mat/0609707]; erratum E76, 019901(E) (2007).

[37] S.G. Samko, A.A. Kilbas and O.I. Marichev, Fractional integrals and derivatives, Gordon and Breach (Amsterdam 1993).

[38] H. Spohn, Phys. Rev. E60, 6141 (1999) [arxiv:cond-mat/9908381].

[39] H. Spohn, J. Stat. Phys. 154, 1191 (2014) [arXiv:1305.6412].

[40] S. Stoimenov and M. Henkel, Symmetry 7, 1595 (2015) [arXiv:1509.00434].

[41] U.C. Täuber, Critical dynamics: a field-theory approach to equilibrium and non-equilibrium scaling behavior, Cambridge University Press (Cambridge 2014).

[42] K.A. Takeuchi and M. Sano, J. Stat. Phys. 147, 853 (2012) [arXiv:1203.2530].

[43] K.A. Takeuchi, J. Stat. Mech. P01006 (2014) [arXiv:1310.0220].

[44] S.Y. Yoon and Y. Kim, Phys. Rev. E68, 036121 (2003).

[45] D.E. Wolf and J. Villain, Europhys. Lett. 13, 389 (1990);

S. das Sarma and P. Tamborenea, Phys. Rev. Lett. 66, 325 (1991).

[46] A. Zoia, A. Rosso and M. Kardar, Phys. Rev. E76, 021116 (2007) [arXiv:0706.1254]. 